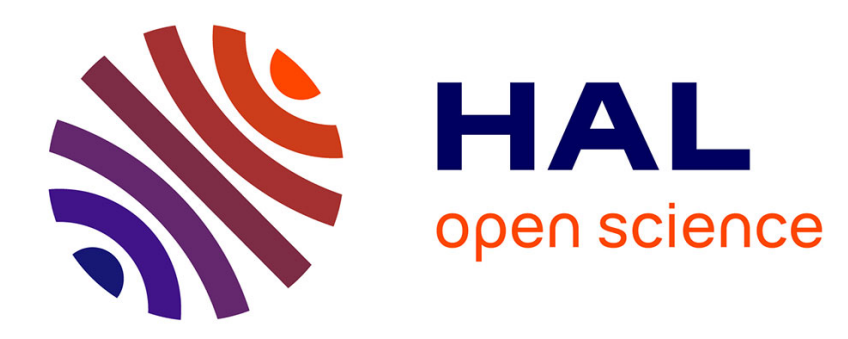

\title{
D'après Machiavel: pour une conception dynamique de la constitution
}

Sébastien Roman

\section{To cite this version:}

Sébastien Roman. D'après Machiavel: pour une conception dynamique de la constitution. Droits, Revue française de théorie, de philosophie et de cultures juridiques, 2014, Sécularisation[s] / 2, 59, pp.231-246. 10.3917/droit.059.0231 . hal-01160898

\section{HAL Id: hal-01160898 \\ https://hal.science/hal-01160898}

Submitted on 8 Jun 2015

HAL is a multi-disciplinary open access archive for the deposit and dissemination of scientific research documents, whether they are published or not. The documents may come from teaching and research institutions in France or abroad, or from public or private research centers.
L'archive ouverte pluridisciplinaire HAL, est destinée au dépôt et à la diffusion de documents scientifiques de niveau recherche, publiés ou non, émanant des établissements d'enseignement et de recherche français ou étrangers, des laboratoires publics ou privés. 


\section{D'APRÈS MACHIAVEL. POUR UNE CONCEPTION DYNAMIQUE DE LA CONSTITUTION}

\section{Sébastien Roman}

\section{Presses Universitaires de France | « Droits »}

2014/1 n 59 | pages 231 à 246

ISSN 0766-3838

ISBN 9782130628880

Article disponible en ligne à l'adresse :

http://www.cairn.info/revue-droits-2014-1-page-231.htm

\section{Pour citer cet article :}

Sébastien Roman, « D'après Machiavel. Pour une conception dynamique de la constitution », Droits 2014/1 (n 59), p. 231-246.

DOI 10.3917/droit.059.0231

Distribution électronique Cairn.info pour Presses Universitaires de France.

(C) Presses Universitaires de France. Tous droits réservés pour tous pays.

La reproduction ou représentation de cet article, notamment par photocopie, n'est autorisée que dans les limites des conditions générales d'utilisation du site ou, le cas échéant, des conditions générales de la licence souscrite par votre établissement. Toute autre reproduction ou représentation, en tout ou partie, sous quelque forme et de quelque manière que ce soit, est interdite sauf accord préalable et écrit de l'éditeur, en dehors des cas prévus par la législation en vigueur en France. Il est précisé que son stockage dans une base de données est également interdit. 
D'APRÈS MACHIAVEL. POUR UNE CONCEPTION DYNAMIQUE DE LA CONSTITUTION

Il est important, avant de se pencher sur la question précise du constitutionnalisme, de donner des éclaircissements sur les raisons de l'actualisation de la pensée machiavélienne, et sur l'interprétation que nous en faisons. Parmi les nombreux travaux philosophiques qui portent sur la place du conflit en démocratie depuis la seconde moitié du $\mathrm{xx}^{\mathrm{e}}$ siècle, le courant néo-machiavélien occupe une place importante. Machiavel fut et continue d'être une source d'inspiration majeure pour repenser la démocratie et définir les conditions de possibilité de son exercice authentique. Cette reprise, de prime abord, peut paraitre paradoxale puisque Machiavel n'était pas un démocrate. Mais elle ne revient pas à répéter sa pensée, abstraction faite des mutations historiques survenues depuis, ni simplement à supprimer d'elle ce qui est anti-démocratique pour l'appliquer à notre temps. Claude Lefort, magistralement, a proposé de l'actualiser par un modèle herméneutique qui consiste à considérer une œuvre non comme un lieu clos ou une forme aux contours bien précis et délimités, historiquement datée, mais comme un espace de questionnement qui se prolonge au-delà de lui-même par ce qu'il donne à penser ${ }^{1}$. L'espace de l'œuvre est indissociable du champ de questionnement qu'elle suscite et des interprétations qui en sont faites. Le prolongement de l'œuvre dépend alors de la possibilité, dans des contextes historiques différents, de s'inspirer de la question qui la traverse. De cette manière, la relecture contemporaine de Machiavel, et sa reprise sous l'angle du républicanisme, s'expliquerait par le contexte historique au lendemain de la seconde Guerre Mondiale. La découverte machiavélienne de la fécondité du conflit civil entre les grands et le peuple à l'occasion de l'étude de la question du principio comment peut naître, se conserver, et prospérer un État ? - s'est révélée très utile pour spécifier et valoriser la démocratie par opposition au totalitarisme. Dans l'effort mené pour combattre la perspective consensualiste du marxisme et son rêve d'une politique sans fard par l'avènement de la société communiste - une société sans classe, harmonieuse et pacifiée, dans laquelle la politique pourrait être transparente - nous avons pu trouver en Machiavel un allié en ce qu'il fait du conflit civil, quand les conditions le permettent, le principe de la vie politique et la condition de la grandeur de l'État. Une telle reprise n'a été possible

1. C. Lefort, Le Travail de l'œuvre Machiavel, Paris, Gallimard, coll. « Bibliothèque de philosophie ", 1972.

Droits - 59, 2014 
que parce que Machiavel lui-même était un pourfendeur des idéologies de son temps, notamment de l'idéologie consensualiste de la tradition ou de l'humanisme civique florentin, lequel célébrait en politique le modèle de la concorde ${ }^{1}$.

Nous avons, de notre côté, en adoptant l'herméneutique lefortienne, proposé une actualisation de la pensée machiavélienne qui met l'accent sur le lien entre les notions de conflit civil et d'imaginaire social, et qui a pour singularité de reprendre la double dimension institutionnelle et extra-institutionnelle du conflit par la tension entre l'idéologie et l'utopie tirée des analyses ricœuriennes de l'imaginaire social moderne ${ }^{2}$. Au fondement-commencement de tout État existe pour Machiavel une opposition contingente entre grands et peuple, entre ceux qui désirent dominer et ceux qui, occupant un statut social inférieur, cherchent à se protéger des puissants. L'homme, par nature, a des désirs insatiables. Sa position sociale fixe son désir et le transforme, selon le rapport de forces effectif, en désir soit de domination, soit de liberté. Mais l'expression du désir reste sujette à la mutabilité. Toute société est composée de parties qui ont chacune tendance à se prendre pour le tout. Le peuple ne lutte contre la domination que par manque d'occasions de goûter à l'insatiabilité des désirs, par habitude de pauvreté, de même que sa lutte contre la domination, si elle devient excessive, peut se transformer en son contraire. Les grands, eux, sont les humains trop humains. Ils reflètent la nature humaine et les tentations liées à l'ambition ou au pouvoir.

Le conflit entre les grands et le peuple, par conséquent, n'est fécond qu'en étant entretenu dans de justes proportions. Machiavel, en son temps, justifiait une régulation des mœurs pour fixer les humeurs - impraticable aujourd'hui qui consistait aussi bien à entretenir la pauvreté du peuple qu'à se servir de l'utilité sociale de la religion et de la guerre pour renforcer respectivement l'obéissance à la loi et l'unité du stato. L'entretien de la figure du commun requiert en supplément la constitution d'un imaginaire politique qui doit reprendre à son compte l'existence d'un imaginaire social. Le souci de la verità effettuale montre que le réel est toujours l'objet de représentations. Il n'y a pas de vie sociale sans des représentations qui la sous-tendent, que le[s] gouvernant[s] doi[ven]t savoir nourrir pour favoriser une entente dans le conflit par la force du mythique et

1. La notion d'humanisme civique florentin mériterait ici d'être travaillée plus précisément.

2. Une nouvelle interprétation de Machiavel depuis les années 1970, que les travaux de Lefort, d'une certaine manière, ont permis d'élaborer, consiste à mettre l'accent sur la notion de conflit civil dans sa pensée dans une perspective post (et non néo)-marxiste. Les analyses de Marie Gaille, Serge Audier, Thierry Ménissier, Laurent Gerbier, John P. McCormick - entre autres - représentent ce nouveau courant. Nous nous inscrivons dans cette perspective, tout en reprenant de Lefort l'indissociabilité des notions de conflit civil et d'imaginaire social dans la pensée machiavélienne, trop peu étudiée aujourd'hui. Voir S. Roman, Conflit civil et imaginaire social. Une approche néo-machiavélienne de la démocratie par l'espace public dissensuel, thèse de doctorat en philosophie réalisée sous la direction de Michel Senellart, ENS de Lyon, 2011, http://tel.archives-ouvertes.fr/tel-00682490 (en cours de réécriture pour une publication en 2016 aux éditions CNRS). L'article proposé ici est tiré de ce travail de doctorat (ibid., pp. 384-460). 
du symbolique. Le prince ne choisit pas de paraître mais doit savoir profiter du caractère nécessairement représentatif de la politique pour bien gouverner. Il est nécessaire d'associer au conflit civil une culture de la gloire de l'État et une valorisation du bene comune, tant du côté du [des] gouvernant[s] pour le[s] convaincre de mettre leur ambition au service de la grandeur de l'Etat en prenant le parti du peuple, que du côté des citoyens pour leur faire croire qu'ils partagent des valeurs identiques, afin qu'ils soient prêts, si nécessaire, à se battre et à mourir ensemble pour elles. Le conflit civil, chez Machiavel, possède une double dimension. Son caractère institutionnel est sa traduction juridique par le modèle de la constitution mixte, indissociable de la dimension extra-institutionnelle du rapport de forces entre le peuple et les grands qui modifie l'attribution de leurs droits respectifs. Les grands, constamment, tentent de profiter de la loi pour assouvir leur désir de domination, ce qui autorise le peuple, quand la nécessité l'impose, à recourir aux armes pour se défendre - le cas-limite étant la guerre civile, qui doit être évitée à tout prix.

Ceci considéré, l'une des grandes difficultés d'une perspective néomachiavélienne pour penser la démocratie aujourd'hui est de savoir comment concevoir la vie politique - et le constitutionnalisme qui doit lui être associé - à partir de la double dimension du conflit civil, tout en prenant en compte les mutations historiques advenues avec la modernité. Un des points cruciaux est le changement majeur survenu avec la Déclaration des droits de l'homme qui engendra une désintrication du droit et du pouvoir ${ }^{1}$. L'imaginaire social n'est plus illusoire aujourd'hui, les hommes ont en commun la faculté de posséder des droits leur permettant de demander des comptes à l'autorité politique. Le pouvoir, désormais, doit être rendu public pour que l'on puisse en évaluer la pertinence. Paul Ricœur, de son côté, sans travailler directement la dimension politique de la Déclaration, en étudie une de ses conséquences historiques par la tension conflictuelle entre l'idéologie et l'utopie qui constitue l'imaginaire social moderne à partir du $\mathrm{XIX}^{\mathrm{e}}$ siècle $^{2}$. Le lien à la fois indissociable et antagoniste entre l'idéologie et l'utopie est tiré d'une praxis sociale conflictuelle opposant des classes. Toute société se caractérise par la mise en place d'un discours dominant, nécessaire pour permettre l'intégration sociale des individus, ainsi que pour construire et enrichir l'identité collective. L'idéologie est également nécessaire pour rendre possible l'exercice du pouvoir en lui apportant la légitimité dont il manque structurellement. L'utopie, a contrario, désigne tout contre-discours qui par son extra-territorialité (la désignation d'un ailleurs), dénonce l'ordre social institué par l'idéologie, ainsi que ce qu'elle exclut et cautionne. La spécificité des sociétés modernes, dès la fin du XIX ${ }^{\mathrm{e}}$ siècle, est de connaître une multiplication des idéologies et des utopies à partir de la fin de l'unité spirituelle du monde : les hommes, ne partageant plus les mêmes valeurs, ont davantage d'occasions d'être en conflit, chacun prétendant avoir raison autant qu'un autre.

1. C. Lefort, "La pensée politique devant les droits de l'homme ", in Le Temps présent, Écrits 1945-2005, Paris, Belin, 2007, p. 417.

2. P. Ricoeur, L'idéologie et l'utopie, (1986), trad. M. Revault d'Allonnes, J. Roman, Paris, Seuil, coll. «La couleur des idées », 1997. 
La tension conflictuelle entre l'idéologie et l'utopie, si on s'inspire de Ricœur, permet d'actualiser adéquatement la pensée machiavélienne. Le modèle machiavélien de l'entente dans le conflit peut être repris par celui contemporain d'un "espace public dissensuel». Une discussion, pour être réussie, ne doit pas nécessairement déboucher sur un accord. Le dissensus, au contraire, peut être fécond. L'espace public dissensuel désigne l'effort de nourrir la politique par l'exercice d'un commun litigieux qui n'est autre qu'une confrontation entre des parties adverses - prioritairement des classes sociales, mais aussi des individus - sur la conception du bien commun à suivre. L'idée, simple, est d'affirmer que le conflit doit être le principe de la vie politique pour permettre l'exercice d'une démocratie authentique, sans cesse réinterrogée dans sa signification et sa finalité.

Comment, dès lors, à partir de tels éléments, concevoir un constitutionnalisme dans une logique néo-machiavélienne? Machiavel était attaché au modèle de la constitution mixte pour des raisons totalement opposées à la tradition. Polybe et Cicéron se trompent sur les raisons qui firent de la constitution mixte la condition de la grandeur de Rome. L'erreur est de condamner les querelles entre les grands et le peuple, de considérer que Rome se perdit en raison des tumultes qui l'agitèrent au lieu de voir en eux le principe de la liberté. L'antagonisme des classes, loin d'être occulté, doit être institutionnalisé et encadré par la loi pour lui donner l'expression la plus juste possible et la plus bénéfique à l'État.

Le constitutionnalisme moderne, ce faisant, a pour grande différence avec le modèle de la constitution mixte de revenir à une conception de la souveraineté absolue et non partagée. La constitution mixte, pour Machiavel, tirait son excellence du partage du pouvoir entre le Sénat et le peuple. Machiavel n'était pas un démocrate; il ne voulait en aucune façon donner le pouvoir au peuple mais le répartir entre les forces antagonistes de la cité pour nourrir le stato de la fécondité du conflit; son républicanisme n'était pas de conviction mais de nécessité. Or la question du partage du pouvoir, aujourd'hui, se pose en termes de séparation et d'équilibre des pouvoirs, sans que soit contredite l'affirmation de la souveraineté absolue du peuple. En revanche, le constitutionnalisme moderne est une technique de limitation de l'autorité étatique qui s'applique au peuple lui-même, reprenant ainsi, d'une certaine façon, la méfiance de Machiavel à son égard. La démocratie suppose que le peuple sache se protéger de sa propre hubris en limitant le pouvoir législatif par un contrôle de constitutionnalité.

Le terme de constitutionnalisme, pour rappel, peut être pris rigoureusement en deux sens. Au sens large, il est une technique de limitation qui reprend deux idées anciennes de la philosophie politique, à savoir la pratique nécessaire d'un gouvernement limité et la substitution d'un gouvernement de la loi à celui des hommes. Ce premier sens permet de "rendre compte de la limitation tant du pouvoir de la Cité (“constitutionnalisme ancien”) que du pouvoir de la royauté par un droit coutumier ("constitutionnalisme médiéval") ${ }^{1}$ ". Au sens restreint

1. O. Beaud, "Constitution et constitutionnalisme ", in Ph. Raynaud, S. Rials (dir.), Dictionnaire de philosophie politique, Paris, Puf, 1998, p. 118. 
- et c'est celui qui importe ici - le constitutionnalisme est une technique de limitation du pouvoir politique appliquée à la figure moderne de l'État. Le constitutionnalisme moderne revient à une conception normative et non descriptive de la constitution ${ }^{1}$. Il est une limitation du pouvoir par le droit, qui va devenir pleinement effectif avec les révolutions américaine et française du XvIII ${ }^{\mathrm{e}}$ siècle, dont les quatre grands principes sont que la " constitution-norme " a pour devoir 1) de fixer les compétences des gouvernants, c'est-à-dire d'organiser «le fonctionnement des pouvoirs publics de l'État $»^{2}$; 2) de protéger les droits fondamentaux individuels de tout abus de pouvoir, y compris du pouvoir législatif par la mise en place d'un contrôle de constitutionnalité ; 3) de veiller à l'équilibre et à la séparation des pouvoirs ; et qu'elle doit, enfin, sur un plan formel, 4) bénéficier elle-même d'un statut exceptionnel en tant que loi suprême, et faire l'objet d'une procédure spéciale de révision pour garantir le principe démocratique de souveraineté populaire, en veillant à ce que personne ne puisse prétendre parler abusivement au nom du peuple. La Constitution, en démocratie, est l'expression du pouvoir constituant du peuple, qui décide de la limitation et de l'exercice des pouvoirs constitués. Elle sert de fondement au système juridique et désigne l'ensemble des règles fondamentales que le peuple a décidé d'établir pour ordonner la vie politique.

Comment, ces précisions données, élaborer un constitutionnalisme dans une perspective néo-machiavélienne de la manière la plus adéquate possible? Quelle figure pourrait bien prendre un constitutionnalisme associé à l'exercice du conflictuel en politique, et à sa double dimension institutionnelle et extrainstitutionnelle? La constitution mixte, chez Machiavel, n'est pas un modèle d'équilibre politique où l'équilibre serait premier, mais d'équilibre précaire obtenu sur fond de déséquilibre perpétuel engendré par la désunion entre le Sénat et le peuple. N'est-ce pas alors une gageure que d'adopter une telle conception de la Constitution? La tension conflictuelle entre l'idéologie et l'utopie, que nous reprenons de Ricœur, inviterait-elle à adopter un constitutionnalisme singulier? Et que pourrait-il être ?

Notre analyse va procéder en deux temps. Trop rares sont les études aujourd'hui qui portent sur la question du constitutionnalisme dans une perspective néo-machiavélienne ${ }^{3}$. D'où l'utilité des travaux de Philip Pettit, lui-même partisan d'une démocratie contestataire dans une perspective républicaine, pour préciser premièrement et comparativement le constitutionnalisme qu'il convient de développer selon le modèle de l'espace public dissensuel. La question, ensuite,

1. Ibid., p. 118.

2. Ibid., p. 119.

3. L'exception majeure est toutefois celle des travaux de McCormick, que nous n'étudions pas ici, de même que nous laissons volontairement de côté l'anti-constitutionnalisme d'Antonio Negri (J. P. McCormick, Machiavellian Democracy, Cambridge U.P., 2011). Voir également M. Gaille-Nikodimov (dir.), Le Gouvernement mixte : de l'idéal politique au monstre constitutionnel en Europe, XIII $-X V I I^{2}$ siècle, Publications de l'Université de SaintÉtienne, coll. «Renaissance et âge classique ", 2005. 
sera celle de la faisabilité de la conception dynamique de la Constitution proposée, eu égard au degré de vertu civique que l'on peut attendre des citoyens.

\section{Le constitutionnalisme de Pettit - ses insuffisances}

Pettit ne s'inspire pas uniquement et directement de Machiavel mais de la tradition républicaine qu'il a l'intention d'approfondir par ses travaux. Il propose une théorie normative du gouvernement dans une perspective républicaine qui définit la liberté comme un état de non-domination, distinct de la noninterférence. Être libre n'est pas seulement pouvoir jouir de ses droits sans subir de contraintes. L'état de non-interférence tolère encore l'existence de dominations arbitraires tant que l'individu en est protégé ou en bénéficie. La liberté, de manière plus exigeante, est de ne pas être sous le pouvoir d'autrui, que ce pouvoir soit effectif ou non. L'originalité de Pettit est de vouloir appliquer cette conception de la liberté aux sociétés contemporaines. L'État doit s'efforcer de protéger tout individu d'une domination arbitraire même potentielle, à laquelle il pourrait être exposé.

L'État en conséquence a le droit d'interférer dans la vie privée des citoyens ou de leur imposer des contraintes en vue du bien commun recherché. Il a le droit d'être coercitif, à condition que les décisions publiques soient jugées légitimes par les citoyens. La maximisation de la non-domination va même jusqu'à justifier des "renoncements ponctuels " à certains de nos droits, ou encore des dispositions constitutionnelles qui, en raison de leur caractère très contraignant, "répugnent à notre sens moral " ${ }^{1}$. Le problème, dès lors, est que la lutte contre la domination privée (dominium) risque de favoriser à son tour une domination exercée par l'État (imperium). D'où les quatre points de la théorie normative pettitienne. Il faut d'une part définir les contraintes constitutionnelles qui doivent encadrer l'exercice du pouvoir; d'autre part, compléter les vertus des institutions par l'institutionnalisation d'un pouvoir de contestabilité dont la finalité sera d'examiner la légitimité des décisions prises; le risque - troisième point - de la corruption chez ceux qui exercent une charge publique suppose de recourir à des mesures de régulation pour rendre viable le projet républicain; la question des mœurs, enfin, est indissociable de l'idéal politique de non-domination, car le projet républicain doit pouvoir s'inscrire dans le cœur des citoyens pour devenir effectif.

Nous nous concentrerons ici sur ce qui, dans ces quatre points, concerne directement le constitutionnalisme. Pettit précise que son constitutionnalisme est avant tout théorique, même s'il s'accorde avec certains principes institutionnels déjà dégagés par la tradition républicaine. Il s'agit, en effet, d'une expérience de pensée qui consiste à imaginer ce que devrait être le constitutionnalisme en lui appliquant le modèle de la liberté comme non-domination. Le point de départ est la formulation de trois contraintes constitutionnelles majeures. Le constitutionnalisme, premièrement, revient à substituer un empire du

1. P. Pettit, Républicanisme : une théorie de la liberté et du gouvernement, trad. P. Savidan, J-F. Spitz, Paris, Gallimard, coll. "Nrf essais », 2003, p. 138. 
droit au pouvoir des hommes, en exigeant que les lois, d'une part, se plient aux contraintes habituelles imposées par l'État de droit (être générales, publiques, compréhensibles), d'autre part qu'elles soient respectées par le gouvernement pour empêcher toute décision politique de devenir arbitraire. Il doit ensuite garantir une "dispersion du pouvoir", au sens d'une séparation classique des pouvoirs qui justifie le contrôle de constitutionnalité des lois par une autorité judiciaire indépendante, mais également le bicamérisme pour se protéger de la tyrannie possible de la majorité, tout en insistant de manière supplémentaire sur l'importance de la décentralisation du pouvoir (cas du fédéralisme aux États-Unis), et sur l'existence de traités internationaux pour contraindre l'État et disperser davantage son autorité ; le constitutionnalisme, enfin, doit protéger les lois les plus fondamentales de la volonté majoritaire. Le texte constitutionnel doit pouvoir être révisé pour être perfectible et s'adapter à l'évolution de la société. En revanche, la procédure de révision, concernant ces lois, doit être rendue complexe pour les protéger d'une simple majorité par des mesures qui pourront varier d'un système juridique à l'autre.

Les trois contraintes constitutionnelles, aussi justes soient-elles, ne permettent pas de parvenir à un constitutionnalisme satisfaisant. Un pouvoir discrétionnaire, nécessaire mais problématique, est laissé aux administrateurs et aux juges (dans l'exécution et l'interprétation des lois) ainsi qu'aux législateurs (dans la formulation de leur contenu). L'originalité de Pettit est de conclure qu'il est nécessaire, pour garantir la démocratie, de compléter le constitutionnalisme envisagé par un pouvoir de contestabilité. Les décisions publiques ne seront légitimes qu'à condition que "nous puissions les reconnaître comme nôtres et nous identifier avec elles [... $]^{1}$ ». La démocratie est affaire de contestabilité et non de consentement.

Pettit, soucieux de la faisabilité de son constitutionnalisme, s'attarde également sur les moyens de garantir son effectivité en travaillant une double question. La première porte sur la vertu civique des citoyens. L'idéal politique de non-domination ne risque-t-il pas d'être irréalisable s'il est au-dessus des forces humaines? Le problème concerne plus particulièrement ceux qui exercent une charge publique. Comment s'assurer qu'ils se consacreront à un tel projet républicain s'ils ne l'apprécient pas eux-mêmes, ou si le pouvoir risque de les corrompre? Pettit considère que la tradition républicaine permet de faire une distinction entre le jugement plutôt optimiste porté sur la nature humaine et celui pessimiste sur les effets du pouvoir. Elle montrerait - Machiavel y compris que l'homme a un désir naturel de gloire qui serait un désir de considération. Tout homme serait soucieux de sa réputation, et s'efforcerait d'être vertueux pour jouir d'une bonne notoriété. L'exercice du pouvoir, toutefois, fait encourir le risque de la corruption car il est certain qu'aucun homme ne passerait avec succès le test de l'anneau de Gygès. Des mesures de régulation sont donc nécessaires, lesquelles prennent la forme de sanctions et de filtres. Le maintien de la république suppose d'utiliser le désir naturel de gloire à des fins politiques, pour 
que la vertu soit encouragée et le vice blâmé. Les sanctions liées à la réputation relèvent du modèle de la main intangible. Tout individu ne pourra empêcher que ses faits et gestes tombent sous le coup d'un jugement par l'opinion publique, "soulignant ainsi l'espèce de pression douce mais constante qu'exerce le désir d'obtenir la considération d'autrui ${ }^{1}$ ". La seconde question que travaille Pettit est celle des mœurs, indispensables pour espérer inscrire la théorie normative du gouvernement dans le cœur des citoyens. Les lois doivent être suppléées par des normes sociales ou habitudes de civilité, grâce auxquelles les citoyens pourront se surveiller entre eux et dénoncer tout comportement illégitime qui entraverait la lutte contre la domination.

Quels sont, maintenant, les points de similitude et de divergence entre le constitutionnalisme pettitien, et celui qui correspond au modèle de l'espace public dissensuel ? L'enjeu, identique, du fait de la perspective républicaine adoptée, est de proposer un constitutionnalisme dont la finalité soit la lutte contre la domination. Les trois contraintes constitutionnelles sont également pertinentes, et s'accordent avec les grands principes du constitutionnalisme moderne.

Les différences avec Pettit sont toutefois nombreuses. On ne peut, premièrement, justifier la maximisation de la non-domination au détriment du droit, sinon pour rendre le républicanisme contraire au respect des libertés fondamentales individuelles, et aggraver le risque de l'imperium de l'État.

Pettit, en second lieu, ne reprend pas de Machiavel le constat anthropologique d'une nature humaine mauvaise, et le cite uniquement pour approfondir la thèse de la corruptibilité par le pouvoir. Or il y a bien une affirmation de la radicalité du mal chez Machiavel. C'est parce que l'homme est naturellement mauvais qu'il peut vouloir abuser du pouvoir qu'on lui accorde, si rien n'est fait pour limiter son désir de domination. L'anthropologie machiavélienne permet de mieux comprendre que la domination est un danger permanent, qui s'étend à tous les domaines de l'existence, de même qu'elle insiste davantage sur la nécessité de dispositifs constitutionnels pour éviter tout abus.

Pettit a beau se méfier de la tyrannie de la majorité et du populisme, on peut également s'inquiéter de les voir ressurgir sous l'espèce de pression douce et constante qu'exerce le désir d'obtenir la considération d'autrui. Une sorte de police citoyenne serait mise en place. Les normes sociales qui favorisent le respect des lois sont par exemple celles qui «demandent aux citoyens de dénoncer les délits dont ils ont connaissance ${ }^{2}$ ".

La théorie de la contestabilité, enfin, se distingue principalement de l'espace public dissensuel par son consensualisme. L'idéal politique de la nondomination, chez Pettit, efface les divisions sociales pour favoriser l'émergence d'une seule classe de vulnérabilité par l'hypothèse d'une faculté d'intercompréhension mutuelle ou de permutabilité des individus. Ces derniers, au-delà de leur appartenance à des groupes sociaux différents, seraient en mesure d'être sensibles à la situation de vulnérabilité de chacun, du fait de leur situation

1. Ibid., p. 301 .

2. Ibid., p. 325. 
d'interdépendance. La théorie de la contestabilité suppose l'existence d'un consensus social possible sur la manière d'évaluer ou de juger la légitimité des décisions publiques par référence à des "raisons civiques". Les raisons civiques sont les normes que tout un chacun reconnaîtrait, qu'il s'agirait d'imposer à l'État pour éviter son imperium. Elles sont obtenues par réflexivité de ce qu'un gouvernement juste doit être, et de ce que signifie respecter la dignité d'autrui. Ainsi, il n'y a pas de conflit civil entre des classes sociales, mais tout au plus des désaccords possibles entre les individus, comparables à des dissensus cognitifs temporaires qu'une discussion réussie surmontera par sa perspective consensualiste. La démocratie contestataire pettitienne ne reprend pas plus la dimension extra-institutionnelle du conflit civil énoncée par Machiavel, qu'elle n'est fidèle à sa dimension institutionnelle en omettant l'antagonisme indépassable des classes sociales.

Il est toutefois vital, comme Pettit le propose, de s'engager en faveur du bicamérisme et du système de proportionnalité, comme de prendre au sérieux le problème du pouvoir des juges et d'opter pour une conception dynamique de la Constitution. Le modèle de l'espace public dissensuel - premier point - justifie de manière semblable le bicamérisme par l'idée que le peuple a sa propre hubris dont il faut se préserver. L'exercice de la majorité, sans contre-pouvoir, est tyrannique. Le système de proportionnalité, quant à lui, ne se justifie pas au nom d'une démocratie inclusive dont la finalité est de permettre à tout un chacun de pouvoir témoigner de la situation de domination dont il serait victime, et de contester les décisions publiques prises. Il est indéniable qu'il doit remplir ces fonctions. Mais l'enjeu, supplémentaire, est que les individus ou groupes sociaux puissent confronter leurs points de vue pour participer à l'exercice d'un commun litigieux. Le système de proportionnalité est au service du conflit civil comme principe politique, au lieu d'être simplement un mode élargi de contestation du gouvernement et de recensement des situations de domination ${ }^{1}$.

Le pouvoir judiciaire - troisième point - est effectivement problématique et ne peut que le demeurer. Les juges, potentiellement, incarnent une nouvelle figure des grands. Pettit propose d'encadrer leur pouvoir par des règles strictes. D'une part, la nécessité de décisions publiques neutres et transparentes s'applique à toute personne exerçant une charge publique. Les juges, d'autre part, doivent être élus par des spécialistes qui seuls sauront les recruter pour leur compétence. Enfin, la pression douce et constante qu'exerce sur eux l'opinion permettra de les contraindre à respecter leurs fonctions. Nous nous accordons uniquement avec Pettit sur les deux premières conditions. On ne peut attendre que les juges soient intègres en se référant au mécanisme de la main intangible.

1. La question supplémentaire, en pratique, est de savoir s'il faut opter pour une représentation proportionnelle intégrale ou partielle à l'Assemblée nationale, ou bien les associer (cas du scrutin mixte) pour contester le scrutin majoritaire. Il faudrait aussi statuer sur le seuil minimal de représentation pour chaque parti ( $5 \%$ ? Plus ? Moins ?), de même qu'il faudrait s'interroger sur la manière de concevoir les circonscriptions législatives pour garantir que le nombre de députés attribué à chacune d'entre elles soit proportionnel au nombre de ses habitants. 
La règle fondamentale est le critère de la compétence qui exige, si on l'applique à la France, la suppression du statut des membres de droit au Conseil constitutionnel. Comment peut-on pleinement garantir l'indépendance des juges et éviter qu'ils n'incarnent une nouvelle figure des grands si on permet aux anciens Présidents de la République d'être membres de droit à vie du Conseil ? N'y a-t-il pas là un risque de voir l'indépendance de la justice entachée d'un lien trop fort avec d'anciennes occupations politiques? L'élection des membres du Conseil constitutionnel pose le problème de l'application du critère de compétence. Qui doit-on reconnaître compétent pour les nommer, afin que les filtres choisis pour leur élection soient effectivement justes et respectés? Les procédures prévues par la Constitution française, sur ce point, sont satisfaisantes. Le Président de la République, le Président du Sénat et le Président de l'Assemblée sont aptes à juger, dès lors que leurs décisions sont soumises à l'avis de la "commission permanente compétente de l'assemblée concernée " (à l'avis des commissions de l'Assemblée et du Sénat dans le cas du Président de la République) ${ }^{1}$. Les membres des commissions aident le Président à faire un choix éclairé. Que la décision prise ne soit pas potentiellement absente d'une stratégie politique n'empêche pas le critère de la compétence d'être retenu.

Le constitutionnalisme enfin doit relever d'une conception dynamique de la Constitution. Pettit le justifie par la nécessité d'adapter la loi à l'évolution de la société pour prendre en compte de nouvelles situations de domination. C'est un point incontestable mais insuffisant. Une conception dynamique de la Constitution est surtout nécessaire pour éviter une séparation trop stricte entre la vie politique et la sphère juridique. Le risque de la constitution-norme, comparativement à la conception institutionnelle comme le souligne Olivier Beaud, est de ne pas considérer suffisamment le droit constitutionnel comme un droit politique. Le danger, en limitant le pouvoir par le droit, est de trop séparer la vie politique de la sphère juridique. La Constitution, au contraire, doit être pensée comme "un "instrument de gouvernement", autant qu'une charte des libertés, et elle est à ce titre inséparable du jeu des forces qui en conditionnent l'application $^{2}$ ». La vie dynamique de l'imaginaire social, par la dialectique conflictuelle de l'idéologie et de l'utopie, rend la Constitution révisable ou évolutive. L'espace public dissensuel a pour projet l'exercice d'une démocratie véritable qui suppose une interrogation perpétuelle des citoyens sur ce qui fonde leur vie commune. Seule une conception dynamique de la Constitution peut permettre une démocratie authentique, en faisant du pouvoir d'auto-institution du peuple une création continuée. Mais l'auto-création continue du peuple doit aussi s'accompagner d'une faculté d'auto-limitation. Il est nécessaire de se protéger de la tyrannie de la majorité et de poser la question de la supraconstitutionnalité. La difficulté est double. Elle est, d'une part, de trouver un juste équilibre entre reconnaître la Constitution comme norme juridique fondamentale et éviter de

1. V. Constitution française du 4 octobre 1958 après la révision de juillet 2008, art. 56, La Documentation française, coll. "Documents d'études ", n 104, 2008, p. 14.

2. P. Avril, Les Conventions de la Constitution : normes non écrites du droit politique, Paris, Puf, coll. "Léviathan ", 1997, p. 4. 
rendre sa procédure de révision trop rigide. D'autre part, elle est de respecter le principe de la souveraineté populaire tout en protégeant la Constitution de toute dérive populiste. Le peuple, bien que souverain, ne saurait avoir tous les droits ${ }^{1}$.

\section{La conception dynamique de la Constitution. À l'épreuve de l'individualisme}

Une telle conception dynamique de la Constitution, aussi juste soit-elle, estelle pour autant praticable ? Peut-on attendre des citoyens qu'ils s'interrogent continuellement sur ce qui fonde leur vie commune ? L'idée soutenue suppose un certain optimisme qui impose de réfléchir à ce que Bruce Ackerman nomme l'économie de la vertu. Ackerman considère que le constitutionnalisme américain, dans son histoire, a pour originalité de développer le modèle de la démocratie dualiste. Les Fédéralistes réussirent à combiner les modèles du monisme et du fondationnalisme, hérités des Européens, par une conception dialectique de la vie constitutionnelle, selon une temporalité double avec d'une part la politique extraordinaire ou constitutionnelle, d'autre part la politique normale ou ordinaire. La dialectique instaurée permet de garder les qualités respectives des deux modèles tout en évitant leurs excès, jusqu'à l'obtention d'un équilibre harmonieux entre démocratie et droits. Monisme et fondationnalisme sont tous deux des conceptions démocratiques par l'affirmation que le pouvoir politique appartient au peuple souverain. Seule diffère la manière de concevoir l'exercice du pouvoir par les représentants. La position moniste revient à dire que tout gouvernement a le droit de parler au nom du peuple à partir du moment où il est élu démocratiquement, jusqu'à refuser - à tort - toute limitation du pouvoir par une Cour constitutionnelle. Les fondationnalistes, au contraire, jugent nécessaire de limiter le pouvoir des représentants au nom de la défense des droits fondamentaux. L'excès, dans ce cas, est de rendre intouchable la Constitution jusqu'à priver, en pratique, le peuple de sa souveraineté.

La force des Fédéralistes est d'avoir su concilier la démocratie avec le droit par une conception dialectique de la vie politique. La politique extraordinaire reprend du monisme l'idée que le pouvoir politique appartient au peuple. C'est la forme supérieure de la vie politique, le moment extraordinaire où le peuple se mobilise pour ré-interroger la Constitution. Il est alors justifié que des représentants, élus populairement par d'irrégulières assemblées, puissent parler au nom du peuple ("We, the People»). La politique extra-ordinaire ne débouche sur une réforme de la Constitution (cas de la législation suprême) qu'à condition que la réforme proposée, initiée par une partie du peuple mobilisée, soit ensuite acceptée par l'ensemble des citoyens. Il revient d'ailleurs à la Cour Suprême d'avertir les citoyens et de faire appel à leur jugement quand

1. La procédure de révision, en France, est fixée par les articles 11 et 89 de la Constitution du 4 octobre 1958. La souveraineté populaire n'est pas confisquée, mais le pouvoir de révision dépend de l'exercice de plusieurs organes (l'exécutif et/ou le législatif). Le problème est de savoir si la souveraineté populaire, par de telles mesures, est suffisamment respectée, notamment quand la révision est adoptée sans référendum. 
un mouvement réformateur cherche à modifier la Constitution. La politique ordinaire, elle, est une limitation du pouvoir des représentants du peuple par la Constitution. Personne ne peut prétendre parler au nom du peuple en temps de politique normale. La Constitution ne le permet qu'à condition que le Congrès, le Président, et la Cour Suprême s'entendent. Les lois proposées par le Congrès doivent être approuvées par le Président et jugées constitutionnelles par la Cour Suprême. Tous les amendements, pour être ratifiés, n'exigent donc pas une politique extraordinaire. Mais le processus d'amendement est bien le moment où la politique constitutionnelle peut avoir lieu, où «l'on peut entendre la voix du corps civique, cette voix certes irrégulière mais rationnelle et guidée par l'esprit civique, qui délibère et tranche des principes fondamentaux, comme elle l'a fait au cours des conventions de l'ère révolutionnaire ${ }^{1}$ ».

L'intention d'Ackerman, par son analyse de la pensée constitutionnelle américaine, est de proposer un néo-fédéralisme. L'histoire constitutionnelle américaine doit être comprise comme une "pratique historique évolutive ", un récit que les Américains élaborent et continuent de développer par une relecture collective de leur Constitution ${ }^{2}$. Ce récit suppose que les Américains entrent en dialogue avec la pensée des Fédéralistes, non pour simplement répéter leur pensée, mais pour la reprendre à leur compte en l'adaptant à leur époque. La force des Fédéralistes est d'avoir su garantir l'exercice d'une démocratie véritable en permettant que le peuple, à des moments extra-ordinaires, puisse directement s'exprimer et soit doté d'une créativité constitutionnelle. Elle est également d'avoir justifié la politique normale, non seulement par la nécessité du mécanisme représentatif, mais par la conscience du peu de vertu civique des citoyens. Les Fédéralistes ont compris qu'il était impossible d'attendre des citoyens un souci permanent de la vie politique. Ils ont proposé une économie de la vertu qui permet de maximiser la vertu civique dont les citoyens sont capables, et qui aboutit aux mesures suivantes : la distinction politique ordinaire/constitutionnelle est nécessaire, car elle prend l'homme tel qu'il est, non tel qu'il devrait être ; le mécanisme représentatif et la vie politique ordinaire en général doivent être encadrés par des contraintes institutionnelles pour forcer les représentants à servir l'intérêt public ; le contrôle de constitutionnalité, enfin, est justifié pour que la Constitution soit protégée des passions et des intérêts de la politique factionnelle.

Le néo-fédéralisme d'Ackerman pose la question majeure suivante. Est-il possible de proposer une conception dynamique de la Constitution qui soit plus accentuée que la sienne, dès lors que le phénomène contemporain de l'individualisme est à prendre en compte? Ackerman, par sa lecture originale de l'histoire constitutionnelle américaine, cherche à redynamiser autant que possible le rapport des Américains à la Constitution en révélant la créativité

1. B. Ackerman, Au nom du peuple : les fondements de la démocratie américaine (1991), trad. J-F. Spitz, Paris, Calmann-Lévy, coll. "Liberté de l'esprit ", 1998, p. 240. L'article $\mathrm{V}$ de la Constitution américaine prévoit la procédure de ratification de l'amendement en temps de politique normale.

2. Ibid., p. 65. 
constitutionnelle que les Fédéralistes leur ont léguée. Les Américains doivent comprendre leur rapport à la Constitution par le modèle narrativiste du récit, qui les autorise à l'interpréter de manière nouvelle aussi bien au niveau de la forme que du contenu, avec la prétention légitime d'être les égaux des pères fondateurs au lieu d'en être des épigones. Leur fidélité doit être dans l'attachement au principe de la démocratie dualiste, sans empêcher leur liberté d'interprétation quant à son expression. Le récit en question est un récit à trois variables, représentées par trois moments révolutionnaires survenus aux époques respectives de la Fondation, de la Reconstruction, et du New Deal ${ }^{1}$. La difficulté, pour que le peuple puisse exercer adéquatement sa puissance créatrice, est de parvenir à faire une synthèse pertinente de ces trois expériences. Les juges, en s'efforçant de faire un tel travail, entrent en relation dialectique avec le peuple en incitant les Américains à s'interroger sur leur Constitution.

La question préliminaire est de savoir s'il est possible de transposer le modèle de la démocratie dualiste au cas français. Le constitutionnalisme allemand, par exemple, est très éloigné du dualisme américain par son fondationnalisme accentué. On peut toutefois penser, comme le souligne Patrick Weil dans sa préface au livre d'Ackerman, que la Constitution française du 4 octobre 1958 permet désormais de concilier le monisme et le fondationnalisme par la création du Conseil constitutionnel. Le monisme hérité du rousseauisme particulier de la Révolution française (la prédominance accordée à la volonté générale) est désormais contrebalancé par l'esprit fondationnaliste qui est à l'origine de la création du Conseil constitutionnel. L'application du modèle de la démocratie dualiste à la France supposerait en supplément de s'interroger sur son histoire constitutionnelle pour savoir quelles sont les expériences de politique extraordinaire qui ont pu la marquer, afin de préciser les modalités d'expression de la créativité constitutionnelle du peuple français.

Mais une question majeure subsiste, qui ne porte pas sur la possibilité mais sur la pertinence d'appliquer le modèle dualiste à la France. Un tel modèle est-il suffisant pour garantir la souveraineté populaire, ou justifie-t-il un équilibre des pouvoirs qui fait la part trop belle aux juges en dissociant trop radicalement la politique normale de la politique constitutionnelle ? Son dualisme, même atténué par la forme dialectique, ne comporte-t-il pas le risque de trop restreindre

1. La politique constitutionnelle a connu trois grands moments révolutionnaires aux États-Unis, qui correspondent tous à des moments où le peuple fut directement sollicité. Le premier, magistral, fut la rédaction par les Fédéralistes d'une nouvelle Constitution des États-Unis en 1787 en se permettant de parler au nom du peuple sans en avoir la légitimité. Le peuple, sollicité pour trancher, l'a ensuite acceptée. Le second moment advint pendant la Reconstruction, soit la période, après la guerre de Sécession, de 1863 à 1877, avec pour événement majeur la ratification du quatorzième amendement. Le dernier moment révolutionnaire eut lieu pendant le New Deal, avec la réélection de F.D. Roosevelt en 1936 qui symbolisa sa victoire sur la Cour Suprême. Ackerman étudie deux autres arrêts de la Cour Suprême au xx ${ }^{e}$ siècle (Brown vs. Board of Education - au sujet de l'égalité raciale - et l'arrêt Griswold vs. Connecticut - au sujet de la liberté sexuelle) et se demande s'ils peuvent être réellement considérés comme "des actes légitimes - et vraiment fondamentaux d'interprétation constitutionnelle" (ibid., p. 179). 
l'exercice direct du peuple, sous prétexte qu'il ne peut en être autrement à cause du peu de vertu civique des citoyens? Christian Nadeau, pour sa part, ne voit aucun inconvénient à traduire la conception machiavélienne du conflit entre grands et peuple par le modèle de la démocratie dualiste. Commentant la constitution dualiste d'Ackerman, il écrit :

Ce qui est à retenir ici est donc l'idée qui est au centre du pluralisme politique : la pluralité axiologique chère au libéralisme moderne demande aux institutions de prévoir les lieux d'expression des visions respectives du bien et de la justice. De manière similaire, la thèse de Machiavel suppose que la vie politique des citoyens est façonnée par leurs institutions, à condition de penser également que les institutions sont elles-mêmes façonnées par les citoyens et, plus encore, par les rapports conflictuels entre les grands et le peuple. Ainsi, le peuple a droit de regard sur des mesures civiles qui peuvent lui être préjudiciables. Mais la loi limite le droit des peuples à agir sur elle et, pour s'assurer de ces limites, bénéficie de l'appui des grands qui ne veulent pas accorder des pouvoirs trop grands au peuple ${ }^{1}$.

Il est vrai que le modèle dualiste s'accorde avec l'idée machiavélienne d'une dialectique entre les institutions et la vie politique, ou d'une redéfinition de la loi selon l'évolution des rapports de forces entre grands et peuple. Le dualisme constitutionnel n'est pas non plus étranger à la perspective républicaine en affirmant que la liberté individuelle suppose un intérêt pour le bien commun ${ }^{2}$. Mais l'accord avec la pensée machiavélienne est-il suffisant? Peut-on accepter que le peuple ne soit convoqué qu'à de rares occasions, qu'il n'intervienne qu'exceptionnellement, sous prétexte que les contraintes institutionnelles en temps de politique normale assurent qu'aucun(e) parti(e) ne puisse parler en son nom ? N'est-ce pas trop faire confiance aux juges dans leur fonction de gardiens de la Constitution? Le dualisme constitutionnel d'Ackerman diffère de l'espace public dissensuel en ce qu'il pose uniquement la question du respect de la souveraineté populaire par ses représentants, ou traite le problème du principe de la souveraineté et de son expression. Les sujets sont le peuple et ses représentants, avec d'un côté le peuple considéré comme un "tout"-le corps politique dans son ensemble ou la population - et de l'autre la vie politique normale et son mécanisme représentatif. Il n'est donc pas question d'un conflit permanent au sein de la vie politique à laquelle participe directement le peuple pour lutter contre la domination.

La théorie de l'économie de la vertu, enfin, est juste mais doit être complétée. Une démarche néo-machiavélienne ne peut que se soucier de prendre l'homme tel qu'il est par souci de la verità effettuale. Il n'est donc plus question d'espérer que l'homme, à l'instar du citoyen grec, soit idéalement dévoué au bien commun. Machiavel, déjà, renonçait à cette idée en prenant en compte le cas de

1. C. Nadeau, "Machiavel : domination et liberté politique ", Philosophiques, 2003, vol. $30, \mathrm{n}^{\circ} 2$, p. 345 .

2. La démocratie dualiste, pour Ackerman, permet de dépasser l'opposition classique entre le libéralisme et le républicanisme en revenant à un "libéralisme républicain " (B. Ackerman, Au nom du peuple, op. cit., p. 60). 
l'ambition. Il se posait déjà la question de l'articulation entre la sphère privée et la sphère publique, et proposait, pour ce faire, de mettre l'ambition au service de la gloire par l'attachement au bene comune. Le problème, depuis, s'est aggravé. L'individualisme est un phénomène majeur des sociétés contemporaines qui affaiblit plus encore la vertu civique des citoyens. Nous l'entendons ici dans le sens tocquevillien d'un phénomène de dépolitisation des individus qui va à l'encontre de leur individualité et menace l'exercice démocratique. Deux points sont essentiels dans l'analyse tocquevillienne de l'individualisme : le premier est la logique de l'intérêt bien entendu, pour combattre l'individualisme ; le second est l'affirmation que l'individualisme est un mal démocratique non nécessaire, dont on peut venir à bout à condition de savoir repolitiser les citoyens ${ }^{1}$.

Mais on ne peut espérer y parvenir en affirmant seulement que la politique normale s'autorégule elle-même par les mécanismes institutionnels prévus par la Constitution. La maximisation de la vertu civique des citoyens doit être envisagée autrement pour devenir effective. Seule l'adoption du conflit comme principe politique peut permettre de donner pleinement vie à la démocratie. L'espace public dissensuel bénéficie d'un ancrage immédiat dans la société, en partant des luttes pour ou contre la domination que mènent les individus et les groupes sociaux. C'est en rendant le commun litigieux que l'on peut intéresser les citoyens à la vie politique, en leur montrant que leurs désaccords peuvent être féconds. L'espoir, de cette manière, est de favoriser davantage leur créativité constitutionnelle que le fait Ackerman.

En conclusion, l'espace public dissensuel, d'inspiration néo-machiavélienne, revient à proposer une conception dynamique de la Constitution, dont le cœur est la tension entre l'idéologie et l'utopie tirée des conflits ou désaccords entre les citoyens. Seul l'exercice d'un commun litigieux permettra de revitaliser aujourd'hui la démocratie, à condition que l'on rende le conflit fécond en favorisant une éthique du dissensus - soit une entente procédurale des citoyens, au minimum, sur les règles qui doivent encadrer leurs désaccords. Cornelius Castoriadis était lui aussi partisan d'une "démocratie ouverte ". Mais on peut regretter qu'il n'ait pas conçu un imaginaire social sur un mode davantage intersubjectif ${ }^{2}$. L'espace public dissensuel propose de le faire sur un mode conflictuel, dans l'espoir de révéler l'homme à sa propre puissance créatrice.

1. Sur l'individualisme, et sa distinction avec l'égoïsme et l'individualité, J.-C. Lamberti, La Notion d'individualisme chez Tocqueville, Paris, Puf, 1970.

2. Sur ce point, cf. J. Habermas, Le discours philosophique de la modernité, (1985), trad. C. Bouchindhomme, R. Rochlitz, Paris, Gallimard, coll. "Bibliothèque de philosophie ", 1988, pp. 387-396 ; D. Martuccelli, "Cornelius Castoriadis : promesses et problèmes de la création ", Cahiers internationaux de sociologie, 2002/2, n 113, pp. 285-305. 


\section{QUADRIGE}
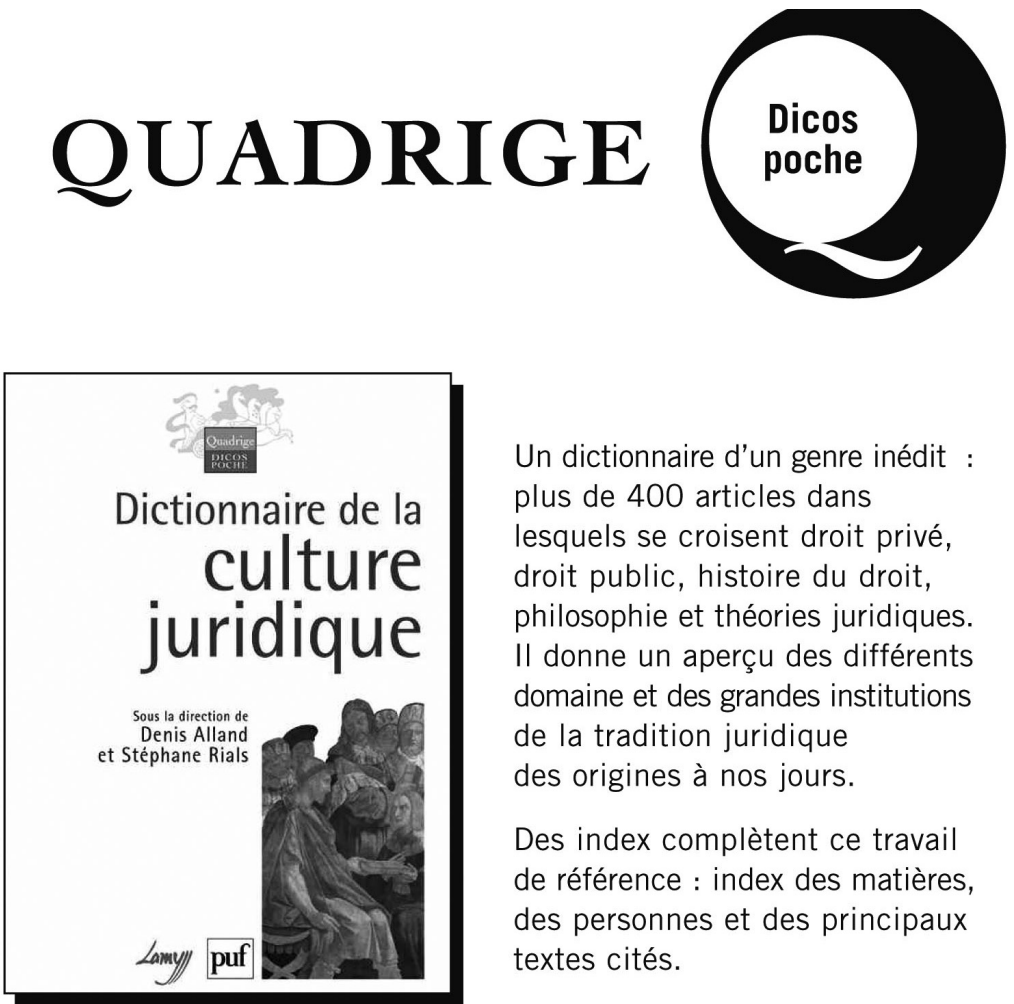

Un dictionnaire d'un genre inédit : plus de 400 articles dans lesquels se croisent droit privé, droit public, histoire du droit, philosophie et théories juridiques. II donne un aperçu des différents domaine et des grandes institutions de la tradition juridique des origines à nos jours.

Des index complètent ce travail de référence : index des matières, des personnes et des principaux textes cités.

Sous la direction de

Denis Alland et Stéphane Rials

- 213 AUTEURS • 409 eNTRÉES •

- 1680 PAgES • 45€ 